\title{
Food-Dependent Exercise-Induced Anaphylaxis: An Overview
}

\author{
Nafisa Husaini Bhagat ${ }^{1}$, Latifa Al Shekaili ${ }^{2}$ and Iman H Nasr ${ }^{3 *}$ \\ ${ }^{1}$ Medical Officer, Clinical Immunology and Allergy Unit, Department of Internal Medicine, The Royal Hospital \\ Sultanate of Oman \\ 2,3Immunologist and allergist, Clinical Immunology and Allergy Unit, Department of Internal Medicine, The Royal \\ Hospital, Sultanate of Oman
}

Received: February 02, 2018; Accepted: March 2, 2018; Published:March 16, 2018

*Corresponding author: Iman H Nasr, Immunologist and allergist, Clinical Immunology and Allergy Unit, Department of Internal Medicine, The Royal Hospital, Sultanate of Oman, Tel: +968-24593056, E-mail: drimannasr@gmail.com

\begin{abstract}
Food-Dependent Exercise-Induced Anaphylaxis (FDEIA) is a rare condition described by the onset of anaphylaxis within a few hours after exposure to food, in association with exercise. Food and exercise is tolerated well independent of one another. Any food can cause FDEIA but the most implicated is wheat. Symptoms may begin at any stage of exercise, in the form of flushing, warmth, urticaria, hypotension and collapse. Diagnosis may be challenging as these symptoms do not necessarily occur each time the individual ingests food and exercises. History is pivotal in making a diagnosis. A careful provocation test combining food and exercise may be required if the diagnosis remains unclear. It is important to raise awareness about this rare entity, so that fatal reactions may be avoided in affected individuals by way of appropriate patient education and prompt management. This article, reports the clinical presentation of two young Omani women who were diagnosed with Food-Dependant Exercise-Induced Anaphylaxis (FDEIA) at our centre. The culprit foods associated with the FDEIA reported here are wheat and chocolate. The clinical manifestations, pathogenesis, diagnostic approach and treatment are elucidated in this article.
\end{abstract}

Key words: Food; exercise; anaphylaxis; IgE mediated; allergy; prick-prick test; epinephrine auto-injector

\section{Introduction}

Food-Dependant Exercise-Induced Anaphylaxis (FDEIA) is a condition described by the onset of anaphylaxis after exposure to food, in association with exercise 1. Interestingly, the culprit food and exercise are independently tolerated by the effected individual 1. The condition is classified as a physical allergy 2. Although, several cases of FDEIA have been reported across the globe it remains uncommon [1, 2, 3]. This case series reports 2 cases of FDEIA where the culprit foods are wheat and chocolate. To the best of our knowledge, the case of Chocolate dependent exercise induced anaphylaxis is the first case to be diagnosed in Oman.

\section{Case 1}

A 24-year-old, young, Omani lady with no prior medical history was referred to our Immunology and allergy service at the Royal Hospital in Oman for an evaluation following an episode of anaphylaxis, where no causative agent was identified. History divulged from the patient revealed that she developed urticarial wheals associated with Dyspnea and ultimately lost consciousness. The symptoms described, manifested almost an hour after she consumed a local patisserie (core ingredients - wheat and cheese) while she was taking a brisk walk. The patient was promptly managed as per the local anaphylaxis protocol at her regional hospital with intramuscular epinephrine, intravenous hydrocortisone and intravenous antihistamines and discharged home once her symptoms had resolved. History revealed that the patient was able to tolerate all the other major food groups (egg, dairy [including cheese], soy, seafood, nuts and peanuts) without any adverse effects. The patient was able to tolerate wheat products (including the aforementioned food item) in the absence of exercise which was the crucial piece of history that leads to the diagnosis of FDEIA.

A retrospective review of the patient's past medical records revealed that the patient had presented with anaphylaxis twice to the hospital after eating a sandwich (core ingredient - wheat). The patient herself recalled engaging in exercise/physical activity a few hours after eating the sandwich, following which her symptoms transpired. Her specific Immunoglobulin E (IgE) to wheat was negative. The specific IgE to the wheat protein omega-5 gliadin was not available.

However, the clinical presentation and patient history was strongly suggestive of wheat dependent exercise induced anaphylaxis (a well reported entity of FDEIA in literature) $[2,4$, 5].

The patient was counselled about avoidance of activity for at least $4-6$ hours following a meal containing wheat as it could potentially trigger anaphylaxis. She was provided with an epinephrine auto-injector as a precautionary measure, in order to promptly abate symptoms of anaphylaxis should she engage in exercise after unknowingly eating a meal that contained wheat. She had no episodes of anaphylaxis since. 


\section{Case 2}

A 26-year-old, young Omani lady with a background of atopic dermatitis (skin biopsy proven) and contact dermatitis to p-Phenylenediamine (PPD) containing hair dyes as demonstrated by patch testing was evaluated at our service for a suspected food allergy. The young lady reported history of recurrent hives associated with occasional Dyspnea and angioedema upon moderate physical activity (mostly when brisk walking but also when climbing a flight of stairs) soon after eating a chocolate snack "on-the-go" but never triggered by exercise or chocolate alone. Her history gave rise to the suspicion of an food-dependent exercised-induced allergy. The suspected foods in this case were plain chocolate bars and chocolate bars containing peanuts.

The patient underwent a prick-prick test to peanut which was negative. This was followed by an oral challenge test to peanuts which yielded a negative result as well. She was then tested for cocoa \& various chocolates by way of prick-prick testing, and subsequently a careful oral challenge which was also negative. Specific immunoglobulin-E (IgE) to cocoa was negative. A challenge test (chocolate followed by exercise in the form of brisk walking) was proved to be negative. At the end of the provocation test, when the patient was discharged she rushed through the hospital corridors and it was that level of physical exertion that triggered a generalised urticarial rash, associated with Dyspnea requiring management with systemic steroids and antihistamines. There by, leading to a diagnosis of a FDEIA (culprit food being chocolate).

\section{Discussion}

Food dependent exercise induced anaphylaxis (FDEIA) is rare 5 . There is limited data estimating the prevalence of the condition. The condition is described as an immunoglobulin E (IgE) mediated allergic reaction triggered by a specific food, only if associated with physical activity/exercise $[1,3,5,6]$. The commonest foods reported with FDEIA are wheat flour and crustacean 5. The clinical presentation of FDEIA may range from urticaria and/or angioedema to anaphylaxis [5, 7].

Making a diagnosis of FDEIA is challenging and it begins with a comprehensive history of events surrounding the episode. A patient presenting with FDEIA would typically present with anaphylaxis that was triggered during or briefly after exercise, only, if the culprit food was ingested a few hours prior to exercising. The patient's ability to tolerate the culprit food and exercise independently without mounting an anaphylactic reaction is vital information that should be elicited from the clinical history. Although the diagnosis of FDEIA is largely dependent on history, objective evidence of sensitization to the culprit food item by way of a challenge test, may further support the diagnosis. Skin testing using either a commercially prepared extract or using fresh food are both accepted modalities of testing for sensitization. Should a skin prick test yield a negative result, then prick-prick testing using fresh food must be performed as not all patient's may yield a positive result with a commercially prepared extract.
This was demonstrated by Hoffmann, et al. while investigating patients with wheat dependent exercise induced anaphylaxis, where only $29 \%$ of testing subjects yielded a positive response to a commercially prepared wheat extract in contrast to $80 \%$ of subjects who yielded a positive response to a freshly prepared wheat flour paste [8]. Alternatively, Immuno CAP commercial assay for specific IgE may be of benefit in demonstrating allergen sensitization, with a reported $80 \%$ sensitivity for wheat (omega- 5 gliadin) which is the protein implicated in wheat dependent exercise induced anaphylaxis [9].

Beyond laboratory and skin prick tests, there is a scope for provocation testing by way of a food-exercise challenge testing. Presently, there is no validated protocol for exercise challenge testing. However, there have been reports on the beneficial role of exercise challenge testing in objectively diagnosing patients with FDEIA $[2,10]$.

The pathogenesis of the disease is not well understood till date. There are some interesting theories in literature that have proposed various physiological changes that occur during exercise and co-factors that may incite a food-dependent exerciseinduced reaction/anaphylaxis.

One such proposed theory suggests exposure to an increased quantity of ingested allergens because of an augmented gastric permeability achieved during exercise [11]. Non-steroidal anti-inflammatory drugs are another recognized cofactor that increases gastric permeability [12]. Thus, history of ingesting non-steroidal anti-inflammatory medications in patients presenting with FDEIA may be valuable in reaching a diagnosis.

Once the diagnosis is reached, it is imperative to educate the patient about the condition. The mainstay of treatment is patient education and avoidance of the allergen in association with exercise. Romano A, et al. suggests avoidance of the sensitized food item for at least 4 hours prior to engaging in any physical activity/exercise [13]. In addition to avoiding the culprit food, advice regarding avoidance of co-factors involved in the pathogenesis of FDEIA such as non-steroidal anti-inflammatory drugs should be offered to the patient.

Epinephrine auto-injector should be prescribed to individuals with FDEIA as anaphylaxis is unpredictable and can occur at any time. Medic-Alert bracelets may be offered if available.

\section{Summary}

As highlighted through this article, FDEIA is an uncommon condition which is yet to be fully understood. A systematic and objective approach, inclusive of a comprehensive history is a must in any patient suspected to have FDEIA to reach a definitive diagnosis.

Education regarding avoidance of the culprit food for at least 4 hours prior to engaging in any form of physical activity, is of paramount importance and should be reiterated to the patient upon each follow up visit. 


\section{Disclaimers}

This article is a brief clinical review of food-dependent exercise-induced anaphylaxis, an entity well reported in literature but has poor awareness among health carers.

\section{References}

1. Du Toit G. Food-dependent exercise-induced anaphylaxis in childhood. Pediatr Allergy Immunol. 2007;18(5):455-463.

2. Aihara $\mathrm{Y}$, Takahashi $\mathrm{Y}$, kotoyori T, Mitsuda T, Ito R, Aihara M et, al. Frequency of food-dependent, exercise induced anaphylaxis in Japanese junior-high-school students. J Allergy Clin Immunol. 2001;108(6):1035-1039.

3. Orhan F, Karakas T. Food-dependent exercise-induced anaphylaxis to lentil and anaphylaxis to chickpea in a 17-year-old-boy. J Investig Allergol Clin Immunol. 2008;18(6):465-468.

4. Kano H, Jui F, Shibuya N, Narita M, Naritaka S, Suko M, et al. Clinical courses of 18 cases with food-dependent exercise-induced anaphylaxis. Arerugi. 2000;49(6):472-478.

5. Beaudouin E, Renaudin JM, Morisset M, Codreanu F, Kanny G, MoneretVautrin DA. Food-dependent exercise-induced anaphylaxis - update and current data. Eur Ann Allergy Clin Immunol. 2006;38(2):45-51.

6. James M Kidd III, Cohen SH, Sosman AJ, Fink JN. Food-dependent exercise-induced anaphylaxis. J Allergy Clin Immunol. 1983;71(4):407411.
7. Maulitz RM, Pratt DS, Schocket AL. Exercise-induced anaphylactic reaction to shellfish. J Allergy Clin Immunol. 1979;63(6):433-434.

8. Hofmann SC, Fischer J, Eriksson C, Bengtsson Gref O, Biedermann T, Jakob T. IgE detection to $\alpha / \beta / \gamma$-glidan and its clinical relevance in wheat-dependent exercise-induced anaphylaxis. Allergy. 2012 Nov;67(11):1457-1460.

9. Matsuo H, Dahlström J, Tanaka A, Kohno K, Takahashi H et, al. Sensitivity and specificity of recombinant omega- 5 gliadin specific IgE measurement for the diagnosis of wheat-dependent exercise induced anaphylaxis. Allergy. 2008;63(2):233-236. doi: 10.1111/j.13989995.2007.01504.x

10. Brockow K, Kneissl D, Valentini L, Zelger O, Grosber M et, al. Using gluten oral food challenge protocol to improve diagnosis of wheatdependent exercise-induced anaphylaxis. J Allergy Clin Immunol. 2015;135(4):977-984. doi: 10.1016/j.jaci.2014.08.024

11. Yano H, Kato Y, Matsuda T. Acute exercise induces gastrointestinal leakage of allergen in lysozyme-sensitized mice. Eur J Appl Physiol. 2002; 87(4-5):358-364.

12. Lichtenberger LM, Zhou Y, Dial EJ, Raphael RM. NSAID injury to the gastrointestinal tract: evidence that NSAIDs interact with phospholipids to weaken the hydrophobic surface barrier and induce formation of unstable pores in membranes. J Pharm Pharmacol. 2006;58(11):1421-1428.

13. Romano A, Di Fonso M, Giufferda F, Papa G, et al. Food-dependent exercise-induced anaphylaxis: clinical and laboratory findings in 54 subjects. Int Arch Allergy Immunol. 2001;125(3):264-272. 